\title{
Electron Spin Dynamics in Semiconductors without Inversion Symmetry
}

\author{
Vadim I. Puller ${ }^{1}$, Lev G. Mourokh ${ }^{1}$, Anatoly Yu. Smirnov ${ }^{2}$ and Norman \\ J.M. Horing ${ }^{1}$ \\ ${ }^{1}$ Department of Physics and Engineering Physics, Stevens Institute of Technology, \\ Hoboken, NJ 07030, USA \\ ${ }^{2}$ D-Wave Systems Inc., 320-1985 W.Broadway, Vancouver, B.C., Canada V6J 4 Y3
}

\begin{abstract}
We present a microscopic analysis of electron spin dynamics in the presence of an external magnetic field for non-centrosymmetric semiconductors in which the D'yakonov-Perel' spin-orbit interaction is the dominant spin relaxation mechanism. We implement a fully microscopic two-step calculation, in which the relaxation of orbital motion due to electron-bath coupling is the first step and spin relaxation due to spinorbit coupling is the second step. On this basis, we derive a set of Bloch equations for spin with the relaxation times $T_{1}$ and $T_{2}$ obtained microscopically. We show that in bulk semiconductors without magnetic field, $T_{1}=T_{2}$, whereas for a quantum well with a magnetic field applied along the growth direction $T_{1}=T_{2} / 2$ for any magnetic field strength.
\end{abstract}

Recent consideration of spin based quantum computation, optical switches, magnetic memory cells, etc. [1] mandates an improved understanding of spin dynamics and, in particular, the spin relaxation rate [2]. The most promising materials for device purposes, III-V and II-VI compounds, have been shown [3] to have spin relaxation rates dominated by the D'yakonov-Perel' (DP) mechanism at moderate temperatures and low hole concentrations, and the spin relaxation time is given by the following semiphenomenological expression [4]:

$$
\frac{1}{\tau_{s}}=q \frac{\alpha^{2}}{\hbar^{2} \varepsilon_{g}} \tau_{p} T^{3}
$$

where $\alpha$ describes conduction band spin splitting due to lack of inversion symmetry (for example $\alpha=0.07$ for GaAs), $\varepsilon_{g}$ is the band gap, $\tau_{p}$ is the average momentum relaxation 
time, and $T$ is the Kelvin temperature $\left(k_{B}=1\right)$. The numerical coefficient $q$ depends on the orbital scattering mechanism.

We develop a fully microscopic theory of spin relaxation in semiconductors, based on a two step analysis of the relaxation process, corresponding to the relaxation time hierarchy involved in (a) electron thermalization due to dissipative bath action, and (b) spin relaxation. In the first stage of solution, we can determine the relaxation rates and fluctuation characteristics of electron orbital motion due to coupling to the bath. Spin relaxation dynamics can be neglected in this stage.

Our analysis of the second stage proceeds with the spin relaxation process due to spinorbit interaction, wherein the orbital degrees of freedom are considered as an effective heat bath, having its characteristics determined in the first stage. A set of Bloch equations having two microscopically determined relaxation times (a longitudinal relaxation time, $T_{1}$, responsible for spin magnetic moment relaxation, and a transverse relaxation time, $T_{2}$, responsible for decoherence) is derived in this second stage. The method of Ref.[5] is employed in both stages of our analysis .

The orbital electron dynamics are determined from operator equations having the form

$$
\begin{aligned}
& \left(\frac{d}{d t}+\gamma_{0}\right) V_{x}(t)+\left(\omega_{c}+\delta\right) V_{y}(t)=\xi_{x}(t), \\
& \left(\frac{d}{d t}+\gamma_{0}\right) V_{y}(t)-\left(\omega_{c}-\delta\right) V_{x}(t)=\xi_{y}(t),
\end{aligned}
$$

and

$$
\left(\frac{d}{d t}+\gamma_{z}\right) V_{z}(t)=\xi_{z}(t),
$$

where $V_{x}(t), V_{y}(t), V_{z}(t)$ are electron velocity operator components $\left(V_{x}=\left(p_{x}-m \omega_{c} y / 2\right) / m\right.$; $\left.V_{y}=\left(p_{y}+m \omega_{c} x / 2\right) / m ; V_{z}=p_{z} / m ;\left[V_{x}, V_{y}\right]_{-}=-i \hbar \omega_{c} / m\right)$, and $\omega_{c}=|e| B / m c$ is the cyclotron frequency. The electron-bath interaction determines the relaxation rates, $\gamma_{0}, \gamma_{z}$, the frequency shift, $\delta$, and the fluctuation sources, $\xi_{x}(t), \xi_{y}(t), \xi_{z}(t)$, involved in the Eq. (2). The Fourier transforms of the velocity correlation functions are given by 


$$
\begin{gathered}
\left\langle\frac{1}{2}\left[V_{x}(\omega) ; V_{x}\right]_{+}\right\rangle=\left\langle\frac{1}{2}\left[V_{y}(\omega) ; V_{y}\right]_{+}\right\rangle=\frac{K_{\perp}(\omega)}{2}\left(\frac{1}{\left(\omega-\omega_{c}\right)^{2}+\gamma_{0}^{2}}+\frac{1}{\left(\omega+\omega_{c}\right)^{2}+\gamma_{0}^{2}}\right) \\
\left\langle\frac{1}{2}\left[V_{x}(\omega) ; V_{y}\right]_{+}\right\rangle=-\left\langle\frac{1}{2}\left[V_{y}(\omega) ; V_{x}\right]_{+}\right\rangle=\frac{K_{\perp}(\omega)}{2 i}\left(\frac{1}{\left(\omega-\omega_{c}\right)^{2}+\gamma_{0}^{2}}-\frac{1}{\left(\omega+\omega_{c}\right)^{2}+\gamma_{0}^{2}}\right)
\end{gathered}
$$

and

$$
\left\langle\frac{1}{2}\left[V_{z}(\omega) ; V_{z}\right]_{+}\right\rangle=\frac{K_{z}(\omega)}{\omega^{2}+\gamma_{z}^{2}},
$$

where

$$
\begin{gathered}
K_{\perp}(\omega)=\int d\left(t-t_{1}\right) e^{i \omega\left(t-t_{1}\right)}\left\langle\frac{1}{2}\left[\xi_{x}(t), \xi_{x}\left(t_{1}\right)\right]_{+}\right\rangle=\int d\left(t-t_{1}\right) e^{i \omega\left(t-t_{1}\right)}\left\langle\frac{1}{2}\left[\xi_{y}(t), \xi_{y}\left(t_{1}\right)\right]_{+}\right\rangle, \\
K_{z}(\omega)=\int d\left(t-t_{1}\right) e^{i \omega\left(t-t_{1}\right)}\left\langle\frac{1}{2}\left[\xi_{z}(t), \xi_{z}\left(t_{1}\right)\right]_{+}\right\rangle,
\end{gathered}
$$

and $[\ldots, \ldots]_{+}$denotes the anticommutator.

In the second stage we analyze spin relaxation due to the DP interaction between spin and electron orbital motion. The corresponding interaction Hamiltonian is given by [4]

$$
H_{D P}=-\sigma_{x} Q_{x}(t)-\sigma_{y} Q_{y}(t)-\sigma_{z} Q_{z}(t)
$$

where

$$
\begin{aligned}
& Q_{x}(t)=-\lambda V_{x}(t)\left(V_{y}^{2}(t)-V_{z}^{2}(t)\right), Q_{y}(t)=-\lambda V_{y}(t)\left(V_{z}^{2}(t)-V_{x}^{2}(t)\right), \\
& Q_{z}(t)=-\lambda V_{z}(t)\left(V_{x}^{2}(t)-V_{y}^{2}(t)\right),
\end{aligned}
$$

and

$$
\lambda=\frac{\alpha m^{3 / 2}}{2 \sqrt{2 \varepsilon_{g}}} .
$$

Employing a second application of the method of Ref. [5] we consider the orbital dynamics in the role of an effective heat bath and obtain a set of Bloch equations for the average spin projections as 


$$
\begin{aligned}
\frac{d}{d t}\left\langle\sigma_{x}(t)\right\rangle & =-\frac{\left\langle\sigma_{x}(t)\right\rangle}{T_{2}}-\left(\omega_{B}+\delta_{x}\right)\left\langle\sigma_{y}(t)\right\rangle, \\
\frac{d}{d t}\left\langle\sigma_{y}(t)\right\rangle & =\left(\omega_{B}+\delta_{y}\right)\left\langle\sigma_{x}(t)\right\rangle-\frac{\left\langle\sigma_{y}(t)\right\rangle}{T_{2}}, \\
\frac{d}{d t}\left\langle\sigma_{z}(t)\right\rangle & =\frac{\sigma_{z}^{0}-\left\langle\sigma_{z}(t)\right\rangle}{T_{1}},
\end{aligned}
$$

where $\sigma_{x}, \sigma_{y}, \sigma_{z}$ are the Pauli matrices and $\sigma_{z}^{0}=-\tanh \left(\hbar \omega_{B} / 2 T\right)$ is the equilibrium $z$ component of spin, $\omega_{B}=g \mu_{B} B / \hbar, \mu_{B}=|e| \hbar / 2 m_{0} c$ is the Bohr magneton, and the $g$-factor depends on material (it is -0.44 for GaAs). Our microscopic determination of the relaxation times $T_{1}$ and $T_{2}$ yields

$$
\frac{1}{T_{1}}=\frac{4}{\hbar^{2}}\left(S_{x x}\left(\omega_{B}\right)+i S_{x y}\left(\omega_{B}\right)\right)
$$

and

$$
\frac{1}{T_{2}}=\frac{2}{\hbar^{2}}\left(S_{x x}\left(\omega_{B}\right)+i S_{x y}\left(\omega_{B}\right)+S_{z z}(0)\right)
$$

where $S_{x x}\left(\omega_{B}\right), S_{x y}\left(\omega_{B}\right)$, and $S_{z z}(0)$ are the Fourier transforms of the correlation functions of the variables $Q_{j}(t),(j=x, y, z)$ of Eq. (8):

$$
S_{i j}(\omega)=\int d\left(t-t_{1}\right) e^{i \omega\left(t-t_{1}\right)}\left\langle\frac{1}{2}\left[Q_{i}(t), Q_{j}\left(t_{1}\right)\right]_{+}\right\rangle
$$

Eq. (8) implies that the spectral functions $S_{i j}(\omega)$ are averages of the sixth power of the electron velocity component operators, and employing Wick's theorem, we obtain

$$
\begin{aligned}
S_{x x}(\omega)= & S_{y y}(\omega)=\lambda^{2}\left\langle\frac{1}{2}\left[V_{x}(\omega) ; V_{x}\right]_{+}\right\rangle\left[\left(\left\langle V_{z}^{2}\right\rangle-\left\langle V_{x}^{2}\right\rangle\right)^{2}+4\left\langle V_{x} V_{y}\right\rangle\left\langle V_{x} V_{y}\right\rangle\right]+ \\
& +\lambda^{2} \int \frac{d \omega_{1}}{2 \pi} \int \frac{d \omega_{2}}{2 \pi} \Xi\left(\omega_{1}, \omega_{2}, \omega-\omega_{1}-\omega_{2}\right) \cdot \\
& \cdot\left\{2\left\langle\frac{1}{2}\left[V_{x}\left(\omega_{1}\right) ; V_{x}\right]_{+}\right\rangle\left\langle\frac{1}{2}\left[V_{x}\left(\omega_{2}\right) ; V_{x}\right]_{+}\right\rangle\left\langle\frac{1}{2}\left[V_{x}\left(\omega-\omega_{1}-\omega_{2}\right) ; V_{x}\right]_{+}\right\rangle+\right. \\
& 2\left\langle\frac{1}{2}\left[V_{x}\left(\omega_{1}\right) ; V_{x}\right]_{+}\right\rangle\left\langle\frac{1}{2}\left[V_{z}\left(\omega_{2}\right) ; V_{z}\right]_{+}\right\rangle\left\langle\frac{1}{2}\left[V_{z}\left(\omega-\omega_{1}-\omega_{2}\right) ; V_{z}\right]_{+}\right\rangle- \\
& \left.-4\left\langle\frac{1}{2}\left[V_{x}\left(\omega_{1}\right) ; V_{x}\right]_{+}\right\rangle\left\langle\frac{1}{2}\left[V_{x}\left(\omega_{2}\right) ; V_{y}\right]_{+}\right\rangle\left\langle\frac{1}{2}\left[V_{x}\left(\omega-\omega_{1}-\omega_{2}\right) ; V_{y}\right]_{+}\right\rangle\right\}, \\
S_{x y}(\omega)=- & S_{y x}(\omega)=\lambda^{2}\left\langle\frac{1}{2}\left[V_{y}(\omega) ; V_{x}\right]_{+}\right\rangle\left[\left(\left\langle V_{z}^{2}\right\rangle-\left\langle V_{x}^{2}\right\rangle\right)^{2}+4\left\langle V_{x} V_{y}\right\rangle\left\langle V_{x} V_{y}\right\rangle\right]+
\end{aligned}
$$




$$
\begin{aligned}
& +\lambda^{2} \int \frac{d \omega_{1}}{2 \pi} \int \frac{d \omega_{2}}{2 \pi} \Xi\left(\omega_{1}, \omega_{2}, \omega-\omega_{1}-\omega_{2}\right) \cdot \\
& \cdot\left\{2\left\langle\frac{1}{2}\left[V_{y}\left(\omega_{1}\right) ; V_{x}\right]_{+}\right\rangle\left\langle\frac{1}{2}\left[V_{y}\left(\omega_{2}\right) ; V_{x}\right]_{+}\right\rangle\left\langle\frac{1}{2}\left[V_{y}\left(\omega-\omega_{1}-\omega_{2}\right) ; V_{x}\right]_{+}\right\rangle+\right. \\
& +2\left\langle\frac{1}{2}\left[V_{y}\left(\omega_{1}\right) ; V_{x}\right]_{+}\right\rangle\left\langle\frac{1}{2}\left[V_{z}\left(\omega_{2}\right) ; V_{z}\right]_{+}\right\rangle\left\langle\frac{1}{2}\left[V_{z}\left(\omega-\omega_{1}-\omega_{2}\right) ; V_{z}\right]_{+}\right\rangle- \\
& \left.-4\left\langle\frac{1}{2}\left[V_{y}\left(\omega_{1}\right) ; V_{x}\right]_{+}\right\rangle\left\langle\frac{1}{2}\left[V_{x}\left(\omega_{2}\right) ; V_{x}\right]_{+}\right\rangle\left\langle\frac{1}{2}\left[V_{x}\left(\omega-\omega_{1}-\omega_{2}\right) ; V_{x}\right]_{+}\right\rangle\right\},
\end{aligned}
$$

and

$$
\begin{aligned}
S_{z z}(\omega)= & \lambda^{2} \int \frac{d \omega_{1}}{2 \pi} \int \frac{d \omega_{2}}{2 \pi} \Xi\left(\omega_{1}, \omega_{2}, \omega-\omega_{1}-\omega_{2}\right) \cdot \\
& \cdot 4\left\langle\frac{1}{2}\left[V_{z}\left(\omega_{1}\right) ; V_{z}\right]_{+}\right\rangle\left\{\left\langle\frac{1}{2}\left[V_{x}\left(\omega_{2}\right) ; V_{x}\right]_{+}\right\rangle\left\langle\frac{1}{2}\left[V_{x}\left(\omega-\omega_{1}-\omega_{2}\right) ; V_{x}\right]_{+}\right\rangle-\right. \\
& \left.-\left\langle\frac{1}{2}\left[V_{x}\left(\omega_{2}\right) ; V_{y}\right]_{+}\right\rangle\left\langle\frac{1}{2}\left[V_{x}\left(\omega-\omega_{1}-\omega_{2}\right) ; V_{y}\right]_{+}\right\rangle\right\}
\end{aligned}
$$

where

$$
\begin{aligned}
\Xi\left(\omega_{1}, \omega_{2}, \omega_{3}\right)= & 1+\tanh \left(\frac{\hbar \omega_{1}}{2 T}\right) \tanh \left(\frac{\hbar \omega_{2}}{2 T}\right)+ \\
& +\tanh \left(\frac{\hbar \omega_{1}}{2 T}\right) \tanh \left(\frac{\hbar \omega_{3}}{2 T}\right)+\tanh \left(\frac{\hbar \omega_{2}}{2 T}\right) \tanh \left(\frac{\hbar \omega_{3}}{2 T}\right)
\end{aligned}
$$

and $\left\langle V_{j}^{2}\right\rangle=\left\langle\frac{1}{2}\left[V_{j}(t) ; V_{j}(t)\right]_{+}\right\rangle=\int \frac{d \omega}{2 \pi}\left\langle\frac{1}{2}\left[V_{j}(\omega) ; V_{j}\right]_{+}\right\rangle,\left\langle V_{x} V_{y}\right\rangle=\left\langle\frac{1}{2}\left[V_{x}(t) ; V_{y}(t)\right]_{+}\right\rangle=$ $\int \frac{d \omega}{2 \pi}\left\langle\frac{1}{2}\left[V_{x}(\omega) ; V_{y}\right]_{+}\right\rangle$

Any model of a bath (phonons, random impurities, etc.) can be accommodated in this general formulation. Even without specifying the nature of the bath, some peculiarities of spin dynamics can be identified. In particular, in the absence of a magnetic field we obtain $T_{1}=T_{2}\left(=\tau_{s}\right.$ of Eq.(1) with the replacement $\left.q \tau_{p} \rightarrow 1 / \gamma_{z}\right)$, as the zero field limit. However, in the presence of a magnetic field $T_{1} \neq T_{2}$. For the case of strong confinement along the z-axis (quantum well with magnetic field in the growth direction) we have $S_{z z}(\omega)=0$ and $T_{1}=T_{2} / 2$ regardless of the magnetic field strength.

In summary, we have derived Bloch equations for electron spins in non-centrosymmetric semiconductors on a fully microscopic basis in the presence of a magnetic field. Explicit expressions for the spin relaxation times have been derived and analyzed using the D'yakonovPerel' spin-orbit interaction mechanism. 
V.I.P., L.G.M. and N.J.M.H. gratefully acknowledge support from the Department of Defense, DAAD 19-01-1-0592. V.I.P. also thanks the organizing committee of MS+S2002 conference for financial support for his participation.

\section{References}

[1] S.A. Wolf, D.D. Awschalom, R.A. Buhrman, J.M. Daughton, S. von Molnár, M.L. Roukes, A.Y. Chtchelkanova, D.M. Treger, Science 294, 1488 (2001); G.A. Prinz, Science 282, 1660 (1998); Y. Nishikawa, A. Tackeuchi, S. Nakamura, S. Muto, and N. Yokoyama, Appl. Phys. Lett. 66, 839 (1995).

[2] W.H. Lau, J.T. Olesberg, and M.E. Flatté, Phys. Rev. B 64, 161301(R) (2001); A. Bournel, P. Dolfus, E. Cassan, and P. Hesto, Appl. Phys. Lett. 772346 (2000); D.M. Frenkel, Phys. Rev. B 4314228 (1991); P.H. Song and K.W. Kim, arXiv:cond-mat/0111076 (2001).

[3] J.M. Kikkawa and D.D. Awschalom, Phys. Rev. Lett. 80, 4313(1998); T.F. Bogges, J.T. Olesberg, C. Yu, M.E. Flatté, and W.H. Lau, Appl. Phys. Lett. 77, 1333 (2000); B. Beschoten, E. Johnston-Halperin, D.K. Young, M. Poggio, J.E. Grimaldi, S. Keller, S.P. DenBaars, U.K. Mishra, E.L. Hu, and D.D. Awschalom, Phys. Rev. B 63 121202(R) (2001).

[4] M.I. D'yakonov and V.I. Perel', Sov. Phys. JETP 33, 1053 (1977); Optical Orientation, Modern Problems in Condensed Matter Science, edited by F. Meier and B.P. Zakharchenya (North-Holland, Amsterdam, 1984), Vol. 8.

[5] G.F. Efremov and A.Yu. Smirnov, Sov. Phys. JETP 53, 547 (1981); L.G. Mourokh and S.N. Zheltov, Physica B 228, 305 (1996); A.Yu. Smirnov, Phys. Rev. E, 56, 1484 (1997); G. Rose and A.Yu. Smirnov J. Phys.: Condens. Matter 13, 11027 (2001). 